\title{
Load dependence of cardiac output in biventricular pacing: Right ventricular volume overload in pigs
}

\author{
David G. Rabkin, MDa \\ Lauren J. Curtis, BA ${ }^{a}$ \\ Santos E. Cabreriza, BA ${ }^{a}$ \\ Alan D. Weinberg, MS ${ }^{b}$ \\ Henry M. Spotnitz, MD ${ }^{\mathrm{a} *}$
}

From the Departments of Surgery and Biostatistics, ${ }^{\mathrm{b}}$ Columbia University College of Physicians and Surgeons, New York, NY.

Supported in part by the National Heart, Lung, and Blood Institute of the National Institutes of Health (R01 HL48109 to H.M.S.) and in part by the Department of Surgery, New York Presbyterian Hospital.

Received for publication Aug 5, 2003; revisions received Dec 10, 2003; accepted for publication Jan 21, 2004.

Address for reprints: Henry M. Spotnitz, MD, Department of Surgery, Columbia University College of Physicians and Surgeons, 622 W 168th St, PH 14-103, New York, NY 10032 (E-mail: hms2@ Columbia.edu).

*George H. Humphrey, II, Professor of Surgery.

J Thorac Cardiovasc Surg 2004;128:98-102

$0022-5223 / \$ 30.00$

Copyright $\odot 2004$ by The American Association for Thoracic Surgery

doi:10.1016/j.jtcvs.2004.01.031
Background: Previous work from our laboratory has demonstrated that optimization of biventricular pacing is load dependent. During acute pulmonary stenosis and right ventricular pressure overload in swine, cardiac output was maximized by pacing the right ventricle $40 \mathrm{~ms}$ before the left ventricle. To extend those studies, this experiment examined biventricular pacing optimization during right ventricular volume overload.

Methods: After median sternotomy in 6 anesthetized domestic pigs, complete heart block was induced by ethanol ablation. A conduit was grafted from the right ventricle to the right atrium to simulate tricuspid insufficiency. During epicardial, atrial tracking DDD biventricular pacing, atrioventricular delay was varied between 60 and $180 \mathrm{~ms}$ in 30-ms increments. Right ventricular-left ventricular delay was varied at each atrioventricular delay from $+80 \mathrm{~ms}$ (right ventricle first) to $-80 \mathrm{~ms}$ (left ventricle first) in 20-ms increments. Aortic flow, right ventricular pressure, and electrocardiogram were measured at each pacemaker setting with the graft clamped and unclamped.

Results: Atrioventricular and right ventricular-left ventricular delays had no significant effect on cardiac output with the graft clamped. With the graft unclamped, however, there was a statistically significant $(P=.003$ by mixed modeling repeated measures analysis of variance) trend toward higher cardiac output with left ventricle-first pacing.

Conclusion: Left ventricle-first biventricular pacing in swine significantly increased cardiac output during acute tricuspid insufficiency but not during the control state. Trials are warranted to develop clinical biventricular pacing for treatment of perioperative right ventricular dysfunction.

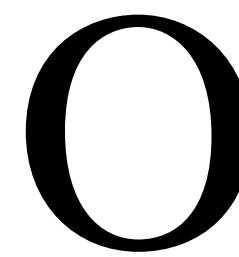

ur laboratory has been examining optimization of biventricular pacing (BiVP) as therapy for acute heart failure in experimental animals. Our hypothesis is that each combination of right ventricular (RV) or left ventricular (LV) pressure or volume overload has specific timing requirements during BiVP for optimization of hemodynamics. Confirmation of this hypothesis would improve understanding of the mechanisms and optimum timing of BiVP in patients and could lead to improved therapy for acute perioperative heart failure.

A previous study from our laboratory of BiVP in swine examined effects of atrioventricular delay (AVD) and interventricular pacing delay (right-left delay, RLD) on cardiac output (CO) in critical pulmonary stenosis with RV pressure overload. ${ }^{1,2} \mathrm{CO}$ was optimized by a positive RLD, the RV paced before the LV. This experiment has extended that study to a different type of heart failure, RV 
volume overload, achieved by simulating tricuspid insufficiency (TI) with an RV-right atrial (RA) conduit in an open-chest porcine model.

Clinical studies demonstrate that BiVP can enhance stroke volume in patients with congestive heart failure ${ }^{3}$ and that the effect is RLD dependent. However, the optimum RLD is highly variable from patient to patient and from time to time in the same patient. ${ }^{4-6} \mathrm{We}$ speculate that this variability reflects variation in right-sided and left-sided pressure or volume overload. Our studies were designed to examine this possibility by evaluating the effect of RLD on stroke volume during isolated forms of heart failure.

\section{Materials and Methods}

All animals received humane care in compliance with "Principles of Laboratory Animal Care" formulated by the National Society for Medical Research and the "Guide for the Care and Use of Laboratory Animals" (http://www/nap.edu/catalog/5140.html). In addition, the experiment was approved by the Institutional Animal Care and Use Committee of Columbia University.

Median sternotomy was carried out in 8 anesthetized domestic pigs (35-45 kg). BiVP with variable RLD was tested with a bipolar RA sensing lead (Medtronic Inc, Houston, Tex) split into two current paths, each connected to a temporary external pacemaker (Medtronic 5388). Bipolar epicardial pacing leads were also placed on the anterior surface of the RV and posterior surface of the LV. Specifically, the RV lead was placed in the center of the anterior $\mathrm{RV}$ toward the RV outflow tract in a vessel-free region. The LV lead was placed by mobilizing the heart anteriorly and suturing the lead to the posterior surface of the LV midway between apex and base. The pacemakers were programmed to minimum ventricular sensitivity (ventricular asynchronous) to prevent inhibition of the second pacemaker by the initial ventricular pacing artifact during BiVP with an RLD greater than 0 .

Once proper function of the pacemaker leads was confirmed, complete heart block (CHB) was established by injection of $0.5-\mathrm{mL}$ aliquots of $100 \%$ ethanol into the region of the bundle of His at the base of the aorta. The cumulative amount of ethanol required to establish CHB ranged from $0.5 \mathrm{~mL}$ to $5 \mathrm{~mL}$. In 2 of the 8 animals, during the attempt to establish third-degree heart block, the RV promptly dilated, systemic blood pressure plummeted, and the hearts showed a fine ventricular fibrillation. One animal could not be cardioverted, and the other appeared globally hypokinetic after electrical cardioversion. These conditions are presumed to reflect intracoronary ethanol injection, and both animals were excluded from data analysis. After CHB was established, a segment of thoracic aorta harvested from another animal and stored in Ringer's lactate and $2 \%$ formalin solution was rinsed and then used as a graft. Modified right-angle aortic arch cannulas $(6.5 \mathrm{~mm}$ Sarns; 3M Health Care, Ann Arbor, Mich) were secured to each end of the graft. The metal cannulas were introduced sequentially through 4-0 Prolene purse-string sutures (Ethicon, Inc, Somerville, $\mathrm{NJ}$ ) first into the RV apex and then, after confirmation of vigorous flow, into the RA. In 1 animal an ultrasound transit-time flow probe (Transonic Systems Inc, Ithaca, NY) placed around the graft confirmed flow, which measured $15 \%$ to $20 \%$ of CO.
The pacing protocol was atrial tracking DDD BiVP. The AVD was varied from 60 to $180 \mathrm{~ms}$ in 30-ms increments. At each AVD the RLD was varied from $+80 \mathrm{~ms}$ (RV first) to $-80 \mathrm{~ms}$ (LV first) in 20-ms increments as described previously elsewhere. ${ }^{1}$ At each pacing setting, ECG, aortic flow, RV pressure, and peripheral arterial pressure were measured in the control state with the graft clamped and during TI with the graft open. This order was not varied between experiments. After data were collected, we again clamped the graft and measured CO to assess the stability of the model. Animals were humanely put to death at the conclusion of the experiment.

\section{Data Analysis}

ECG, RV pressure, and aortic flow velocity were sampled at 200 $\mathrm{Hz}$ with a 16-channel analog to digital converter (MacLab; ADInstruments Inc, Milford, Mass) and recorded on a digital computer (Power Macintosh 7100/66; Apple Computer, Inc, Cupertino, Calif). CO was determined for each experimental phase by integrating aortic flow velocity over time during a single complete respiratory cycle free of arrhythmia. A statistical modeling procedure (described in the following section) was used to determine the effects of heart rate, AVD, and RLD on CO. To determine the effect of AVD on CO, the RLD was kept at 0 and the AVD was changed while the aortic flow velocity was recorded. Heart rate was calculated from the average R-R interval of 5 beats free of ectopy.

\section{Statistical Analysis}

Changes in CO across various levels of AVD were modeled with the PROC MIXED procedure in the SAS software suite (SAS Institute, Inc, Cary, NC). Because repeated measurements within an animal may be correlated, this procedure allows one to model this correlation structure, commonly referred to as a covariance pattern. This allows improved estimates of the standard errors of measurement and thus more powerful tests. There are a number of covariance structures available. Three of the more common ones are compound symmetry, for correlations that are constant for any two points in time, autoregressive order one, for correlations that are smaller for time points further apart, and unstructured, which has no mathematic pattern within the covariance matrix. Other covariance structures that are usually tested include the Toplitz and the heterogeneous compound symmetry structure. A likelihood ratio test or a procedure known as the Akaike information criterion is used to discern which covariance pattern allows the best fit. ${ }^{7}$

\section{Results}

Average $\mathrm{CO}$ at the conclusion of the experiments $(1.76 \pm$ $0.18 \mathrm{~L} / \mathrm{min}$ ) was not significantly different from the beginning of the experiment after the induction of $\mathrm{CHB}$ and insertion of the RV-RA conduit $(1.75 \pm 0.18 \mathrm{~L} / \mathrm{min})$. This $\mathrm{CO}$, however, was significantly lower than that measured immediately after opening the chest $(2.6 \pm 0.14 \mathrm{~L} / \mathrm{min})$. There were no statistically significant effects of AVD on $\mathrm{CO}$ in either control or TI states (Figure 1). However, although $\mathrm{CO}$ peaked in the middle of the examined range of AVD for control, there was a statistically insignificant trend towards higher $\mathrm{CO}$ at higher AVDs for TI. There were no 


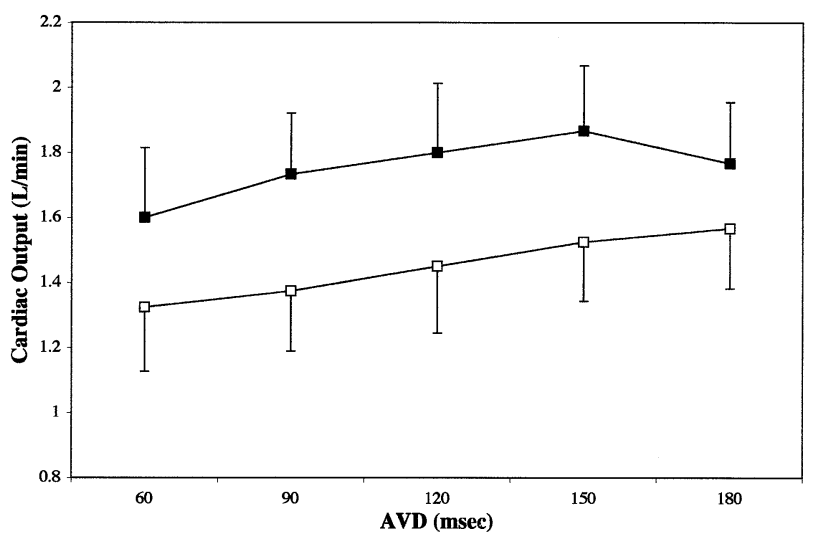

Figure 1. Relationship between $C O$ and AVD in control state (filled squares, $n=6$ ) and TI (open squares, $n=6$ ). Error bars represent SE. Differences in CO across range of AVDs were not statistically significant for either control or $\mathrm{TI}$.

significant changes in heart rate within each experimental state (control and TI), although induction of TI resulted in increases in heart rate relative to control $(83.1 \pm 3.36$ beats/min vs $73.5 \pm 4.00$ beats/min; Table 1 ).

The average effect of RLD on $\mathrm{CO}$ is presented in Figure 2, $A$. In the control state, there was no statistically significant effect. During TI, CO improved significantly with negative RLD. The optimal RLD during TI was negative (LV pacing first) in 5 animals and positive $(+40 \mathrm{~ms}, \mathrm{RV}$ first) in 1 (Figure 2, B). There were no apparent experimental differences in the animal that responded differently. $\mathrm{CO}$ at the optimal RLD of $-20 \mathrm{~ms}(1.5 \pm 0.14 \mathrm{~L} / \mathrm{min})$ was associated with a $9.5 \%$ improvement in CO relative to the RLD of 0 (1.37 \pm 0.13 $\mathrm{L} / \mathrm{min})$, but this change was not statistically significant $(P$ $=.09$ ). In Figure 3, $\mathrm{CO}$ is plotted against the offset from the optimum RLD, again demonstrating that small changes in RLD can alter CO by more than $10 \%$ in this model.

\section{Discussion}

These results demonstrate that BiVP with optimized RLD in swine ameliorates depressed RV function in the setting of acute TI. The data provide additional evidence that optimized timing of BiVP in the setting of acute heart failure is load dependent. Enhanced ventricular function is reflected in $\mathrm{CO}$ and stroke volume. RLD optimization alone increases CO $10 \%$ relative to simultaneous BiVP $(\mathrm{RLD}=0)$. Previous studies by other investigators have demonstrated that BiVP enhances stroke volume without increasing myocardial energy metabolism, ${ }^{5,6}$ in contrast to inotropic agents, which increase myocardial energy demand.

This study did not examine the importance of lead location relative to RLD. That will be the subject of a future
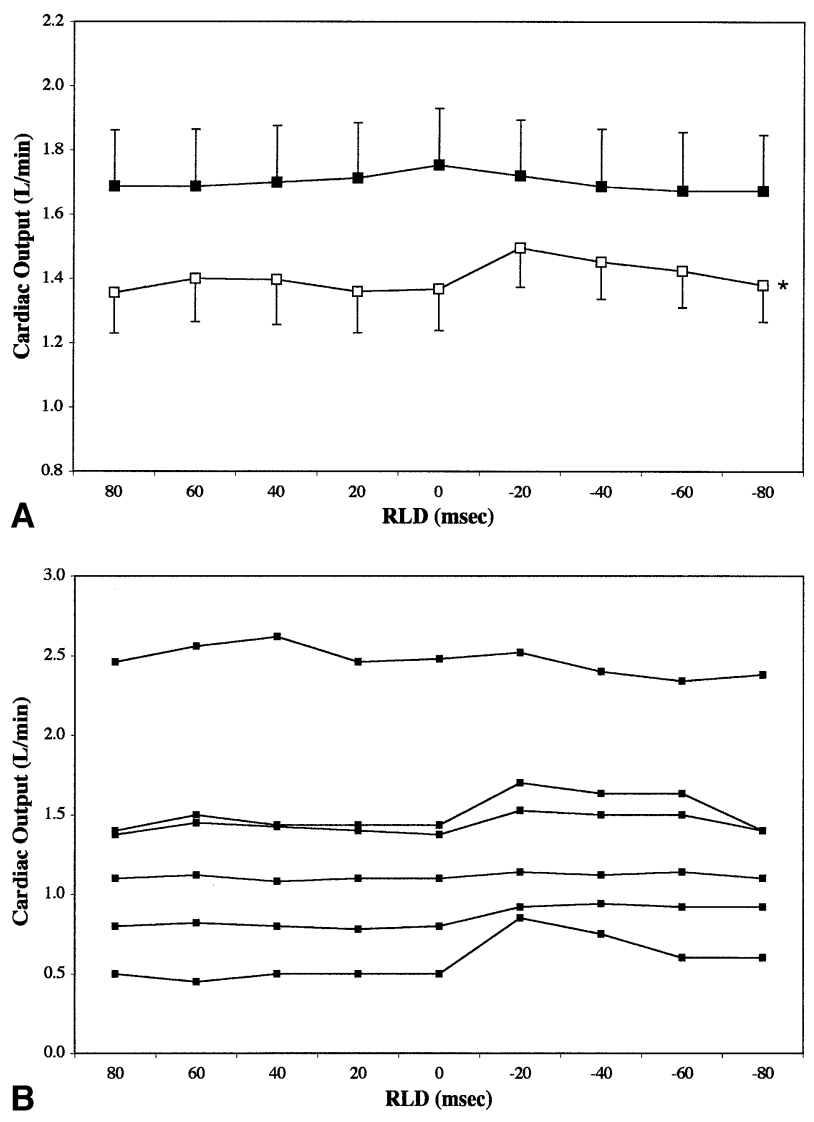

Figure 2. A, Relationship between CO and RLD in control state (filled squares, $n=6$ ) and $\mathrm{TI}$ (open squares, $\mathrm{n}=6$ ). Error bars represent SE. Differences in CO across range of RLD were not statistically significant during control state, but during $\mathrm{TI}$ negative RLD significantly improved $\mathbf{C O}(\boldsymbol{P}=.0031$ by mixed modeling repeated measures analysis of variance, asterisk). B, Relationship between $\mathrm{CO}$ and RLD during $\mathrm{TI}$ at each animal's optimum AVD.

study. In addition, the use of a valved conduit to simulate TI may have produced regional dysfunction of the anterior RV that is not present clinically.

Our previous study of pulmonary stenosis demonstrated that $\mathrm{CO}$ increased $20 \%$ during optimized pacing versus simultaneous BiVP. ${ }^{1}$ Optimum RLD in that model of RV pressure overload was $+40 \mathrm{~ms}$, the RV paced before the $\mathrm{LV}$. In this experiment, LV-first BiVP provided the maximum $\mathrm{CO}$, but the improvement versus simultaneous BiVP was smaller than in our previous study and the timing was less reproducible.

Thus 1 of our 6 animals in TI responded differently than the others despite normal baseline hemodynamics. This variability may reflect problems with our model, including possible ethanol-related myocardial injury, injury to the RV during grafting of the RV-RA conduit, or inconsistent regurgitant flow through the conduit despite our attempts to 
TABLE 1. Heart rates at different time points during the experiment

\begin{tabular}{lcccc}
\hline Animal & Baseline & After CHB & Control & TI \\
\hline 1 & 80 & 70 & 71 & 92 \\
2 & 83 & 81 & 70 & 82 \\
3 & 92 & 91 & 90 & 93 \\
4 & 74 & 70 & 75 & 81 \\
5 & 81 & 76 & 75 & 80 \\
6 & 85 & 61 & 60 & 71 \\
Mean & 82.5 & 74.8 & 73.5 & 83.1 \\
SEM & 2.43 & 4.23 & 4.00 & 3.36 \\
\hline
\end{tabular}

standardize the degree of TI. There was consistent depression of $\mathrm{CO}$ after establishment of CHB and insertion of the RV-RA conduit, suggesting a possible generalized injury. Our conclusions concerning the effect of RLD on CO during TI remain valid, however, because whatever problems were present affected the control state as well. Despite less compelling results, this experiment advances our previous work to a new form of acute heart failure and increases the evidence that optimized timing during BiVP reflects pathologic loading conditions.

Interestingly, although there was no statistically significant effect of AVD on CO during TI, there was a trend towards improved output at higher AVDs. It appears likely that reduction of $\mathrm{CO}$ in this study reflects RV failure and that effects of AVD and RLD are mediated by changes in RV function. Meisner and colleagues ${ }^{8}$ defined optimal AVD as the interval allowing the atrioventricular valves to remain open long enough to maximize ventricular filling without permitting atrioventricular regurgitation.

Our publications to date have not defined mechanisms for the effects of AVD and RLD on CO and have not examined mechanistic differences between effects in pulmonary stenosis or TI. AVD is believed to act through effects on ventricular filling, whereas RLD is more likely to influence critical aspects of the synchrony of contraction. Timing is likely to have important effects on end-diastolic volume in TI because of the critical balance between antegrade and retrograde flow. On the other hand, in critical pulmonary stenosis it is likely that the RV is maximally distended by afterload mismatch, and RV-first pacing may provide advantages by increasing the duration of effective systolic ejection. Measurements of atrial filling pressures as well as RV and LV pressures, volume, shape, and wall motion are critical to understanding these issues. Our preliminary data indicate that optimized RLD improves LV symmetry throughout the cardiac cycle. ${ }^{2}$

Studies of the mechanism of optimization of BiVP are clearly needed. Such studies should define geometric changes by means of echocardiography, sonomicrometry, or conductance. These methods have been used to advantage

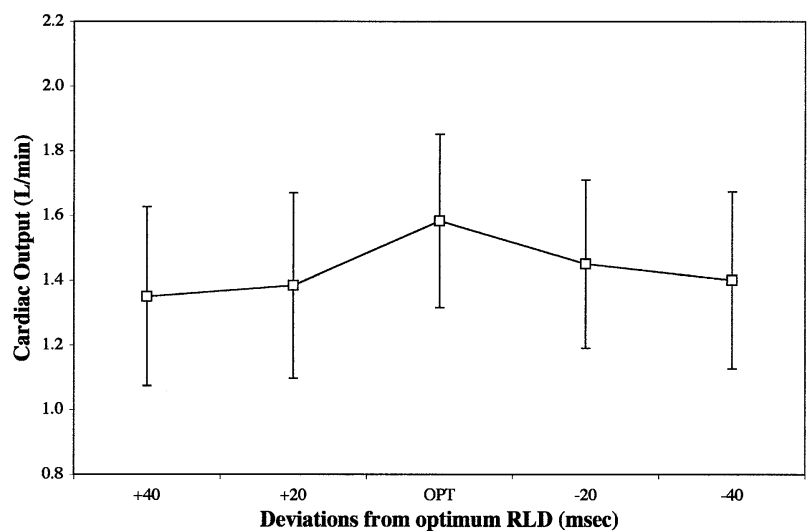

Figure 3. Relationship between $\mathrm{CO}$ and deviation from optimal RLD during TI. Error bars represent $S E, n=6$. This figure maximizes incremental effect of optimum RLD timing by eliminating differences in absolute timing of optimum RLD. Effect of error of $20 \mathrm{~ms}$ in optimum RLD timing is $10 \%$ decrease in $\mathrm{CO}$.

by our laboratory in studies of pulmonary stenosis. ${ }^{2,9}$ Myocardial oxygen consumption would also be an interesting subject of future research.

Our results provide insight into potential avenues for clinical development of BiVP. Our previous studies in pulmonary stenosis indicate not only that RV first pacing is beneficial, but also that properly timed BiVP is better than either RV or LV pacing alone. These results in TI also indicate that BiVP is preferable to LV or RV single-site pacing, with LV pacing preceding RV pacing for optimum benefit. Although acute studies in laboratory animals with normal myocardium may not be directly transferable to the clinical environment, accumulating data suggest that patients in acute heart failure are likely to benefit from BiVP rather than single-site LV or $\mathrm{RV}$ pacing. ${ }^{10}$ The best results are likely to be obtained with timing optimized for each patient. Clinical studies are needed to confirm this hypothesis.

\section{References}

1. Rabkin DG, Cabreriza SE, Curtis LJ, Mazer SP, Kanter JP, Weinberg $\mathrm{AD}$, et al. Load dependence of cardiac output in biventricular pacing: right ventricular pressure overload in pigs. J Thorac Cardiovasc Surg. 2004;127:1713-22.

2. Rabkin DG, Cabreriza SE, Curtis LJ, Spotnitz HM. Effect of variable interventricular delay on LV geometry during biventricular pacing; pulmonary stenosis in pigs [abstract]. ASAIO. 2003;49:174.

3. Abraham WT. Rationale and design of a randomized clinical trial to assess the safety and efficacy of cardiac resynchronization therapy in patients with advanced heart failure: the Multicenter InSync Randomized Clinical Evaluation (MIRACLE). J Card Fail. 2000;6:369-80.

4. Barold SS. What is cardiac resynchronization therapy? Am J Med. 2001;111:224-32

5. Ukkonen H, Beanlands RS, Burwash IG, de Kemp RA, Nahmias C, Fallen E, et al. Effect of cardiac resynchronization on myocardial efficiency and regional oxidative metabolism. Circulation. 2003;107: 28-31. 
6. Nelson GS, Berger RD, Fetics BJ, Talbot M, Spinelli JC, Hare JM, et al. Left ventricular or biventricular pacing improves cardiac function at diminished energy cost in patients with dilated cardiomyopathy and left bundle-branch block. Circulation. 2000;102: 3053-9.

7. Akaike $\mathrm{H}$. A new look at the statistical model identification. IEEE Trans Auto Control. 1974;AC-19:716-23.

8. Meisner JS, McQueen DM, Ishida Y, Vetter HO, Bortolotti U, Strom JA, et al. Effects of timing of atrial systole on LV filling and mitral valve closure: computer and dog studies. Am J Physiol. 1985;249(3 Pt 2):H604-19.

9. Rabkin DG, Cabreriza SE, Curtis LJ, Quinn TA, Weinberg AD, Hordof $\mathrm{AD}$, et al. Mechanisms of optimized biventricular pacing in pulmonary stenosis: effects on left ventricular geometry in swine. $P A C E$. In press.

10. Zimmerman FJ, Starr JP, Koenig PR, Smith P, Hijazi ZM, Bacha EA. Acute hemodynamic benefit of multisite ventricular pacing after congenital heart surgery. Ann Thorac Surg. 2003;75:1775-80.

\section{ON THE MOVE?}

\section{Send us your new address at least six weeks ahead}

Don't miss a single issue of the journal! To ensure prompt service when you change your address, please photocopy and complete the form below.

Please send your change of address notification at least six weeks before your move to ensure continued service. We regret we cannot guarantee replacement of issues missed due to late notification.

\section{JOURNAL TITLE:}

Fill in the title of the journal here.

\section{OLD ADDRESS:}

Affix the address label from a recent issue of the journal here.

\section{NEW ADDRESS:}

Clearly print your new address here.

Name

Address

City/State/ZIP

\section{COPY AND MAIL THIS FORM TO:}

Elsevier Inc.

Subscription Customer Service

6277 Sea Harbor Dr

Orlando, FL 32887
OR FAX TO:

407-363-9661

OR E-mail:

elspcs@elsevier.com

\section{OR PHONE:}

800-654-2452

Outside the U.S., call

407-345-4000 